\title{
RCE-4, a potential anti-cervical cancer drug isolated from Reineckia carnea, induces autophagy via the dual blockade of PI3K and ERK pathways in cervical cancer CaSki cells
}

\author{
WEI XIANG ${ }^{1}$, REN-JING ZHANG ${ }^{2}$, GUI-LAN JIN ${ }^{3}$, LI TIAN ${ }^{1}$, FAN CHENG $^{1}$, JUN-ZHI WANG ${ }^{1}$, \\ XIANG-FEI XING ${ }^{3}$, WEI XI ${ }^{3}$, SHU-JUN TANG ${ }^{4}$ and JIAN-FENG CHEN ${ }^{1}$ \\ ${ }^{1}$ Hubei Key Laboratory of Natural Products Research and Development, College of Biological and \\ Pharmaceutical Sciences, China Three Gorges University, Yichang, Hubei 443002; ${ }^{2}$ Department of Pathology, \\ Affiliated Hospital of North Sichuan Medical College, Nanchong, Sichuan 637000; ${ }^{3}$ The First People's Hospital of Yichang \\ and The People's Hospital of China Three Gorges University, Yichang, Hubei 443002, P.R. China; \\ ${ }^{4}$ Department of Biomedical Engineering, Illinois Institute of Technology, Chicago, IL 60616, USA
}

Received April 24, 2019; Accepted August 29, 2019

DOI: $10.3892 /$ ijmm.2019.4389

\begin{abstract}
The steroidal saponin RCE-4 (1 $\beta, 3 \beta, 5 \beta$, 25S)-spirostan-1, 3-diol 1-[ $\alpha$-L-rhamnopyranosyl- $(1 \rightarrow 2)-\beta$ D-xylopyranoside], isolated from Reineckia carnea, exerts significant anti-cervical cancer activity by inducing apoptosis. The potential effect of RCE-4 on proliferation inhibition and autophagy induction has rarely been studied. Therefore, the focus of the present study was to investigate the effects of RCE-4 on proliferation, and to elucidate the detailed mechanisms involved in autophagy induction in cervical cancer cells. CaSki cells were treated with RCE-4 or/and autophagy inhibitors, and the effect of RCE-4 on cellular proliferation was assessed by MTT assay. The pro-autophagic properties of RCE-4 were subsequently confirmed using monomeric red fluorescent protein-green fluorescent protein-microtubule-associated proteins 1A/1B light chain 3B (LC3) adenoviruses and CYTO-ID autophagy assays, and by assessing the accumulation of lipid-modified LC3 (LC3II). The mechanisms of RCE-4-induced autophagy were investigated by western blot analysis. The results demonstrated that inhibiting autophagy significantly promoted RCE-4-induced cell death, indicating that autophagy served a protective role following RCE-4 treatment. In addition, RCE-4-induced autophagy was reflected by increased expression levels of the serine/threonine-protein
\end{abstract}

Correspondence to: Professor Jian-Feng Chen, Hubei Key Laboratory of Natural Products Research and Development, College of Biological and Pharmaceutical Sciences, China Three Gorges University, 8 University Avenue, Yichang, Hubei 443002, P.R. China E-mail: chenjianfeng2003@126.com

Key words: cervical cancer, $(1 \beta, 3 \beta, 5 \beta, 25 \mathrm{~S})$-spirostan-1, 3-diol 1-[ $\alpha$-L-rhamnopyranosyl-( $1 \rightarrow 2)-\beta$-D-xylopyranoside], autophagy, AMP-activated protein kinase, PI3K/Akt/mTOR pathway, Ras/Raf/ MEK/Erk pathway kinase ULK1, phosphorylated (p)-ULK1, p-Beclin-1 and LC3II, the formation of autophagosomes and autolysosomes, and sequestosome 1 (p62) degradation. Subsequent analysis indicated that RCE-4 activated the AMP-activated protein kinase (AMPK) pathway by upregulating AMPK and p-AMPK, and also inhibited the PI3K and extracellular signal-regulated kinase (ERK) signaling pathways by downregulating p-PI3K, p-Akt, p-mTOR, Ras, c-Raf, p-c-Raf, dual specificity mitogen-activated protein kinase kinase (MEK)1/2, p-MEK1/2 and p-Erk1/2. Additionally, with increased treatment times RCE-4 may impair lysosomal cathepsin activity and inhibit autophagy flux by suppressing the expression of AMPK, p-AMPK, ULK1, p-ULK1 and p-Beclin-1, and upregulating that of $\mathrm{p} 62$. These results indicated that the dual RCE-4-induced inhibition of the PI3K and ERK pathways may result in a more significant anti-tumor effect and prevent chemoresistance, compared with the inhibition of either single pathway; furthermore, dual blockade of PI3K and ERK, and the AMPK pathway may be involved in the regulation of autophagy caused by RCE-4. Taken together, RCE-4 induced autophagy to protect cancer cells against apoptosis, but AMPK-mediated autophagy was inhibited in the later stages of RCE-4 treatment. In addition, autophagy inhibition improved the therapeutic effect of RCE-4. These data highlight RCE-4 as a potential candidate for cervical cancer treatment.

\section{Introduction}

Natural products are valuable resources for the screening of anti-cervical cancer drugs, and include compounds such as artesunate (1), resveratrol (2), betulinic acid (3) and Timosaponin A-III (4). Reineckia carnea (Liliaceae), a traditional Chinese herb containing the saponin $(1 \beta, 3 \beta, 5 \beta$, 25S)-spirostan-1, 3-diol 1-[ $\alpha$-L-rhamnopyranosyl- $(1 \rightarrow 2)-\beta$ D-xylopyranoside] (RCE-4; Fig. 1A) as a biologically active component, has been used to treat diseases including rheumatism, coughs and hepatitis $(5,6)$. To the best of our knowledge, the steroidal saponins primarily exert anti-inflammatory 
and antitumor activity, with toxicity towards both normal and tumor cells (7). However, RCE-4 exhibited a significant anti-inflammatory (8) and notable cytotoxic effects on cancer CaSki, HeLa, HT-29 and CNE-2 cell lines, whereas it exerted relatively weak cytotoxic effects in normal Marc-145 and MDCK cells (9). Furthermore, CaSki cell xenograft experiments using nude mice demonstrated that RCE-4 inhibited tumor growth and had notably low levels of toxicity in normal tissues, including those of the liver and the uterus (10).

Drug-induced tumor-cell death is a complex process that includes various modes of programmed cell death, including apoptosis, autophagy and necrosis (11). Cross-talk has been observed between autophagy (type II programmed cell death), a process in which newly formed membrane-encapsulated vesicles phagocytose and consume cellular components including damaged organelles and misfolded protein aggregates, and apoptosis, which share various cell death mediators, such as Bcl-2 and Beclin-1 (12). Normally, autophagic self-degradation not only removes damaged organelles and misfolded proteins $(13,14)$, but also recycles nutrients. However, deregulation of autophagy may induce tumor cell death, and may therefore serve as a cell survival pathway to inhibit apoptosis, either in combination with apoptosis or as a secondary mechanism when the former is ineffective (15-17). Mechanistically, numerous studies have shown that the PI3K/AKT and Ras/Raf/dual specificity mitogen-activated protein kinase kinase (MEK)/ERK pathways serve an important role in autophagy by inhibiting the expression of mTOR, which is a key homeostatic regulator of cellular proliferation (18). In addition, the AMP-activated protein kinase (AMPK) signaling pathway, which is closely associated with energy metabolism, has also been indicated to activate autophagy (19-21).

Conversely, cross-talk between the Ras/Raf/MEK/Erk and PI3K/Akt pathways in regulating tumor cell proliferation, differentiation, apoptosis and senescence has been identified. This cross-talk may provide the focus for further studies investigating drug therapies that inhibit both signaling networks (22-24). Specifically, activation of the PI3K/Akt pathway promotes cell survival by inhibiting cell cycle progression; additionally, high activity levels of Erk, which is regulated by the Ras/Raf/MEK/Erk pathway, promote cyclin D1 expression and cellular proliferation. Moreover, activation of the PI3K/Akt or Ras/Raf/MEK/Erk pathways inhibits apoptosis by phosphorylating and blocking apoptosis-associated targets including Bcl-2, Bad and caspase-9 $(3,25)$. These studies indicate that the ERK and the PI3K pathways promote cell proliferation and survival. However, inhibitors of each single pathway have limited anti-tumor activity, as the inhibition of one pathway results in the compensatory activation of the other (18). This may also partially explain why cancer treatment often results in resistance to chemotherapeutic drugs. For example, the MEK inhibitor PD98059 blocks the ERK pathway, leading to an abnormal increase in AKT activation levels (26). In addition, in breast cancer cells, the activity of an PI3K inhibitor was demonstrated to be enhanced, resulting in a decreased level of proliferation and enhanced anti-tumor activity by co-treatment with an ERK pathway inhibitor (27). These examples suggest that simultaneous inhibition of the ERK and PI3K pathways in the treatment of certain tumor cells may contribute to the improved anti-tumor activity of chemotherapeutic drugs.
In previous studies, RCE-4, as a potential anti-cervical cancer chemotherapy drug, was demonstrated to induce mitochondria-mediated apoptosis, which was considered to be the mechanism for inhibiting the proliferation of CaSki and HeLa cells $(5,9)$. The aim of the present study was to clarify whether RCE-4-induced autophagy is a survival or death mechanism during cervical cancer treatment. Subsequently, the levels of autophagy were assessed by treating cells with different concentrations of RCE-4, and to determine the degree of autophagy at different treatment exposure times. Given the key roles of the AMPK, PI3K and ERK pathways in cancer cell proliferation and autophagy, it was necessary to additionally identify whether RCE-4 treatment affected the expression and phosphorylation statuses of proteins involved in these pathways. Collectively, the aim of the present study was to improve the understanding of the anti-tumor mechanisms of RCE-4.

\section{Materials and methods}

Reagents and antibodies. The RCE-4 preparation used in the present study was isolated from $R$. carnea and its structure is demonstrated in Fig. 1A. A 50-mM stock solution of purified RCE-4 was prepared in DMSO, and diluted to the desired concentration with RPMI-1640 medium prior to use. Rabbit monoclonal antibodies against $\beta$-actin (cat. no. 4970), AMPK $\alpha$ (cat. no. 5831), phosphorylated (p)-AMPK $\alpha$ (cat. no. 2535), the serine/threonine-protein kinase ULK1 (ULK1; cat. no. 8054), p-ULK1 (cat. no. 14202), Beclin-1 (cat. no. 3495), p-Beclin-1 (cat. no. 14717), microtubule-associated proteins 1A/1B light chain 3B (LC3; cat. no. 12741), sequestosome 1 (p62; cat. no.8052), mTOR (cat. no. 2983), p-mTOR (cat. no. 5536), PI3K (cat. no. 4257), p-PI3K (cat. no. 4228), Akt (cat. no. 4691), p-Akt (cat. no. 4060), Ras (cat. no. 3339), c-Raf (cat. no. 9422), p-c-Raf (cat. no. 9431), MEK1/2 (cat. no. 8727), p-MEK1/2 (cat. no. 9154), p-Erk1/2 (cat. no. 4370) and Erk1/2 (cat. no. 4695), all purchased from Cell Signaling Technology, Inc., were used at a dilution of 1:1,000. The horseradish peroxidase-labeled secondary antibody (anti-rabbit IgG), which was purchased from Cell Signaling Technology, Inc. (cat. no. 7074V), was used at a dilution of 1:5,000. The CYTO-ID Autophagy Detection kit (cat. no. ENZ-51031) was purchased from Enzo Life Sciences Inc., and the autophagy inhibitors bafilomycin A1 (Baf A1), 3-methyladenine (3-MA) and chloroquine (CQ) were obtained from MedChemExpress. The monomeric red fluorescent protein (mRFP)-green fluorescent protein (GFP)-LC3 tandem fluorescent protein kit was purchased from Han heng Biotechnology Co., Ltd.

Cell culture. The human cervical cancer CaSki cell line was obtained from the Cell Bank of the Shanghai Institutes for Biological Sciences, Chinese Academy of Sciences. The cells were cultured in RPMI-1640 medium (Gibco; Thermo Fisher Scientific, Inc.) containing $10 \%$ fetal bovine serum (FBS: Gibco; Thermo Fisher Scientific, Inc.) and 0.2\% HEPES at $37^{\circ} \mathrm{C}$ and $5 \% \mathrm{CO}_{2}$.

Growth inhibitory assays. CaSki cells $\left(2 \times 10^{4}\right.$ cells/well) were seeded into 96 -well plates, incubated at $37^{\circ} \mathrm{C}$ for $12 \mathrm{~h}$ and subsequently treated with $1,2,4,6$ and $8 \mu \mathrm{M} \mathrm{RCE}-4$ for an additional $48 \mathrm{~h}$. For the autophagy inhibition assay, 
the CaSki cells were pretreated with the selective autophagy inhibitors 3-MA (8 mM), CQ $(30 \mu \mathrm{M})$ and Baf A1 $(0.4 \mu \mathrm{M})$ for $6 \mathrm{~h}$, and then treated with RCE-4 $(0,1,2,4,6$ and $8 \mu \mathrm{M})$ for an additional $48 \mathrm{~h}$. Subsequently, $20 \mu \mathrm{l} 5 \mathrm{mg} / \mathrm{ml}$ MTT (Sigma-Aldrich; Merck KGaA) reagent was added to each well, and the cells were cultured for another $4 \mathrm{~h}$; the medium was gently removed, and the adherent cells were lysed in $150 \mu \mathrm{l}$ DMSO solution for 10 min with shaking. The absorbance at $490 \mathrm{~nm}$ was measured using a microplate reader (Tecan Group, Ltd.), and the inhibition rate was calculated according to the following formula: Inhibition rate $=[1$ - optical density (OD) value of the administration group/OD value of blank control group] x $100 \%$.

CYTO-ID autophagy detection assay. The CYTO-ID Autophagy Detection kit was used to selectively label pre-autophagosomes, autophagosomes and autolysosomes; bright fluorescence is detectable in autophagic vacuoles, thereby providing a quantitative method for autophagy detection (28). The CYTO-ID autophagy detection assay was performed according to the manufacturer's kit protocol. CaSki cells were treated with RCE-4 at concentrations of 0 , 8,12 and $16 \mu \mathrm{M}$ for 6,12 and $24 \mathrm{~h}$ each. Following the collection of the adherent cells and those in solution, the cells were resuspended in $1 \mathrm{X}$ assay buffer (provided by the CYTO-ID Autophagy Detection kit) and centrifuged (1,000 x g) at room temperature for $5 \mathrm{~min}$ to remove residual supernatant impurities, including cell growth medium and serum. After carefully removing the buffer, cells were suspended and stained with $500 \mu 1$ of $0.1 \%(\mathrm{v} / \mathrm{v})$ CYTO-ID Green staining solution (1 $\mu$ l CYTO-ID Green Detection Reagent diluted with $1 \mathrm{ml}$ $1 \mathrm{X}$ Assay buffer) for $30 \mathrm{~min}$ at room temperature in the dark. After treatment, stained cells were collected via centrifugation and washed with $1 \mathrm{X}$ Assay Buffer. The cell pellets were then resuspend in $500 \mu \mathrm{l}$ of fresh $1 \mathrm{X}$ Assay Buffer to analyze autophagy status. To detect autophagic vesicles, the green fluorescence emission $(530 \mathrm{~nm})$ of $\geq 1 \times 10^{4}$ cells was detected using flow cytometry (BD FACSVerse; BD Biosciences), and the data were analyzed using BD FACSuite ${ }^{\mathrm{TM}}$ software (version 1.0.0.1477; BD Biosciences).

Assessment of autophagic flux. To determine the autophagy levels in living cells, mRFP-GFP-LC3 adenoviruses or plasmids have been widely used to identify autophagosomes and autolysosomes $(29,30)$. However, this method is only able to indicate a specific point within the process, rather than the autophagic status of the cells at different points following drug treatment. Following culture of the mRFP-GFP-LC3 adenovirus-transfected CaSki cells in cell culture dishes (Wuxi NEST Biotechnology Co., Ltd.) for $6 \mathrm{~h}$ at a multiplicity of infection of 200, $12 \mu \mathrm{M}$ RCE-4 was added for an additional 6, 12, 24 or $30 \mathrm{~h}$. The fluorescence values of the green dots, indicating autophagosomes, and red dots, indicating autophagosomes and autolysosomes, were observed using laser confocal fluorescence microscopy (magificatin, x1,000; LCFM; Olympus FV1200; Olympus Corporation). The number of red and yellow dots (indicating autophagosomes) in the merged images was then recorded.

Western blot analysis. CaSki cells were exposed to RCE-4 at a concentration of $0,8,12$ and $16 \mu \mathrm{M}$ for 6,12 or $24 \mathrm{~h}$. Following incubation, the cells lysed in radioimmunoprecipitation assay buffer (containing 1\% phosphorylated protease inhibitor and $1 \% \mathrm{PMSF})$ were centrifuged $(12,000 \mathrm{x} \mathrm{g})$ at $4^{\circ} \mathrm{C}$ for $10 \mathrm{~min}$, and the total protein was quantified using a bicinchoninic (BCA) protein concentration acid assay kit (Beyotime Institute of Biotechnology). The protein samples were adjusted to a uniform concentration with $5 \mathrm{X}$ buffer and water, and denatured by boiling at $100^{\circ} \mathrm{C}$ for $10 \mathrm{~min}$. The proteins $(60 \mu \mathrm{g} / \mathrm{well})$ were separated using SDS-PAGE with a $6-15 \%$ polyacrylamide gel, and transferred onto a PVDF membrane (EMD Millipore). Following blocking at room temperature for $2 \mathrm{~h}$ with $5 \%$ skim milk, the membrane was incubated with the corresponding primary antibodies at $4{ }^{\circ} \mathrm{C}$ overnight. The membrane was washed 5 times with TBS $+0.05 \%$ Tween-20 (TBST) and the secondary antibody was subsequently added prior to further incubation for $1.5 \mathrm{~h}$ at $37^{\circ} \mathrm{C}$. An enhanced chemiluminescent kit (Beyotime Institute of Biotechnology) was used to visualize the protein bands, and the results were recorded with Kodak Film or a Luminescence Imaging System (Tanon 5200; Tanon Science and Technology Co., Ltd.). The grey values were analyzed with $\beta$-actin as a loading control. Densitometry was analyzed using Image $\mathbf{J}$ version 2.1 software (National Institutes of Health).

Statistical analysis. The data are expressed as the mean \pm standard deviation. GraphPad prism v.5.0 (GraphPad Software, Inc.) was used for all statistical analyses, and the differences between the treatment groups and control group were assessed by one-way analysis of variance followed by Dunnett's post hoc tests. $\mathrm{P}<0.05$ was considered to indicate a statistically significant difference.

\section{Results}

Autophagy inhibition increases the level of RCE-4-induced CaSki cell death. To investigate the effects of RCE-4 on the growth of human cervical cancer cells, an MTT assay was performed using CaSki cells. The results revealed that RCE-4 treatment significantly decreased the viability of CaSki cells after $48 \mathrm{~h}$ of treatment, which was compared with an RCE-4-untreated control group (Fig. 1B). In addition, the RCE-4-induced inhibition was enhanced following pretreatment with the autophagy inhibitors 3-MA, CQ or Baf A1 for $6 \mathrm{~h}$. The half maximal inhibitory concentration values were $4.18,3.36,3.32$ and $1.54 \mu \mathrm{M}$ for the RCE-4 control, the 3-MA+RCE-4, the CQ+RCE-4 and the Baf A1+RCE-4 groups, respectively. It was demonstrated that the inhibition of autophagy significantly sensitized CaSki cells to RCE-4-induced cell death, inferring that RCE-4 activated autophagy in anti-cervical cancer cells (Fig. 1B).

RCE-4 triggers autophagy in cervical cancer cells. Using the CYTO-ID autophagy assay, mRFP-GFP-LC3 tandem fluorescent protein kit and western blot analysis, RCE-4 was determined to induce autophagy in CaSki cells. The CYTO-ID autophagy assay kit was used to demonstrate the presence of autophagosomes in RCE-4 treated CaSki cells; the results indicated an increase in the formation of autophagosomes following treatment with 8,12 and $16 \mu \mathrm{M}$ RCE-4 for 6,12 and $24 \mathrm{~h}$ (Fig. 2A). 
A

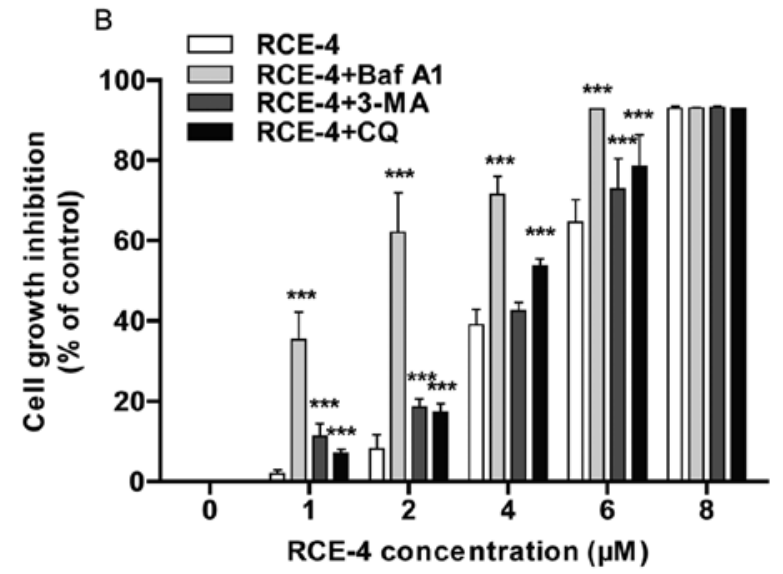

Figure 1. Autophagy inhibitors 3-MA, CQ and Baf A1 enhance the ability of RCE-4 to inhibit CaSki cell proliferation. (A) Chemical structure of RCE-4. (B) CaSki cells were treated with RCE- 4 for $48 \mathrm{~h}$, following pretreatment with autophagy inhibitors for $6 \mathrm{~h}$, and proliferation inhibition was assessed by MTT assay. Values for each RCE-4 concentration were generated from 3 independent experiments, and are presented as the mean \pm standard deviation. ${ }^{* * *} \mathrm{P}<0.001$ vs. control group (the corresponding concentration of RCE-4 without autophagy inhibitor treatment). 3-MA, 3-methyladenine; CQ, chloroquine; Baf A1, bafilomycin A1; RCE-4, (1 $\beta, 3 \beta, 5 \beta, 25 \mathrm{~S})$-spirostan-1, 3-diol 1-[ $\alpha$-L-rhamnopyranosyl-(1-2)- $\beta$-D-xylopyranoside].

A

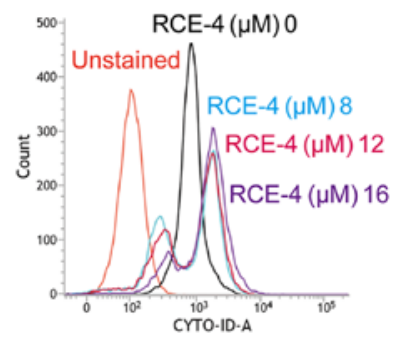

B
Control
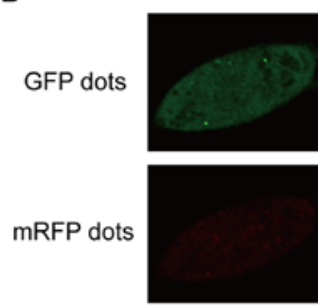

Overlay

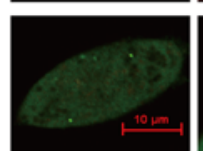

$12 \mathrm{~h}$

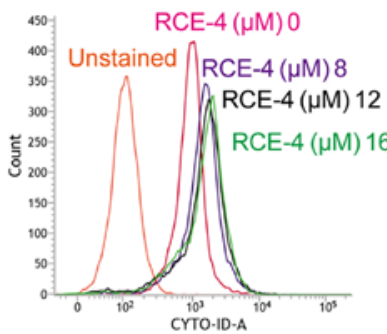

$6 \mathrm{~h}$
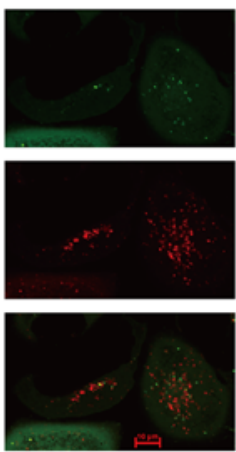

$12 \mathrm{~h}$
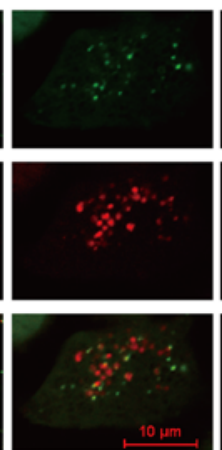

$24 \mathrm{~h}$

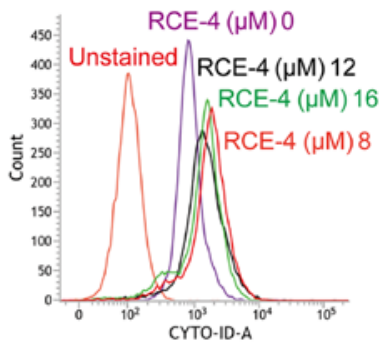

$24 \mathrm{~h}$

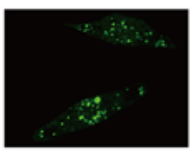

$30 \mathrm{~h}$
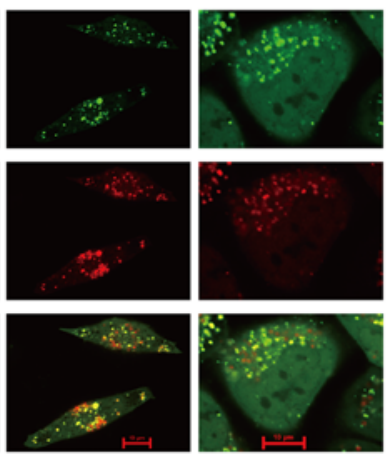

C

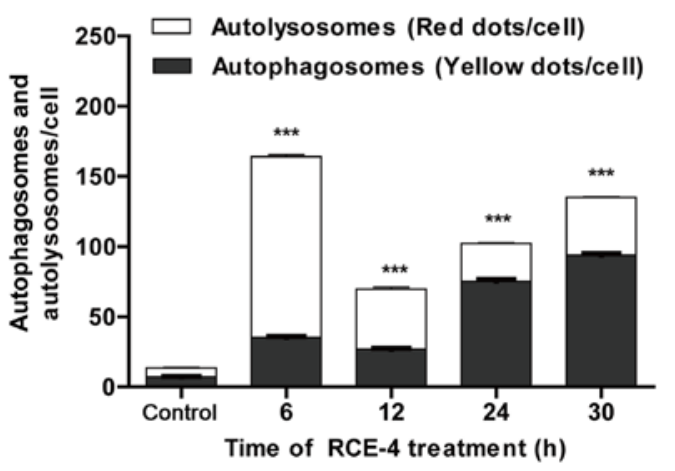

Figure 2. RCE-4 promotes autophagy in CaSki cells. (A) CYTO-ID histograms of RCE-4-treated cells indicated a shift to the right in comparison with the RCE-4-untreated group (control group) after an exposure period of 6, 12 or $24 \mathrm{~h}$, indicating an increase in the mean fluorescence intensity of the RCE-4-treated group. (B) Cells were transfected with mRFP-GFP-LC3 adenoviruses for $6 \mathrm{~h}$, and treated with RCE-4 for a further $6,12,24$ or $30 \mathrm{~h}$. The formation of autophagosomes (yellow dots) and autolysosomes (red dots) in each cell was observed and quantified by laser confocal fluorescence microscopy. A total of 50 cells for each condition were counted. Scale bars=10 $\mu \mathrm{m}$. (C) Changes in the number and percentage of autophagosomes and autolysosomes in the control and RCE-4-treated groups are presented at different RCE-4 treatment times. ${ }^{* * * *} \mathrm{P}<0.001$ vs. RCE-4-untreated group. GFP, green fluorescent protein; mRFP, monomeric red fluorescent protein; RCE-4, $(1 \beta, 3 \beta, 5 \beta, 25 \mathrm{~S})$-spirostan-1, 3-diol 1-[ $\alpha$-L-rhamnopyranosyl-( $1 \rightarrow 2)-\beta$-D-xylopyranoside]. 

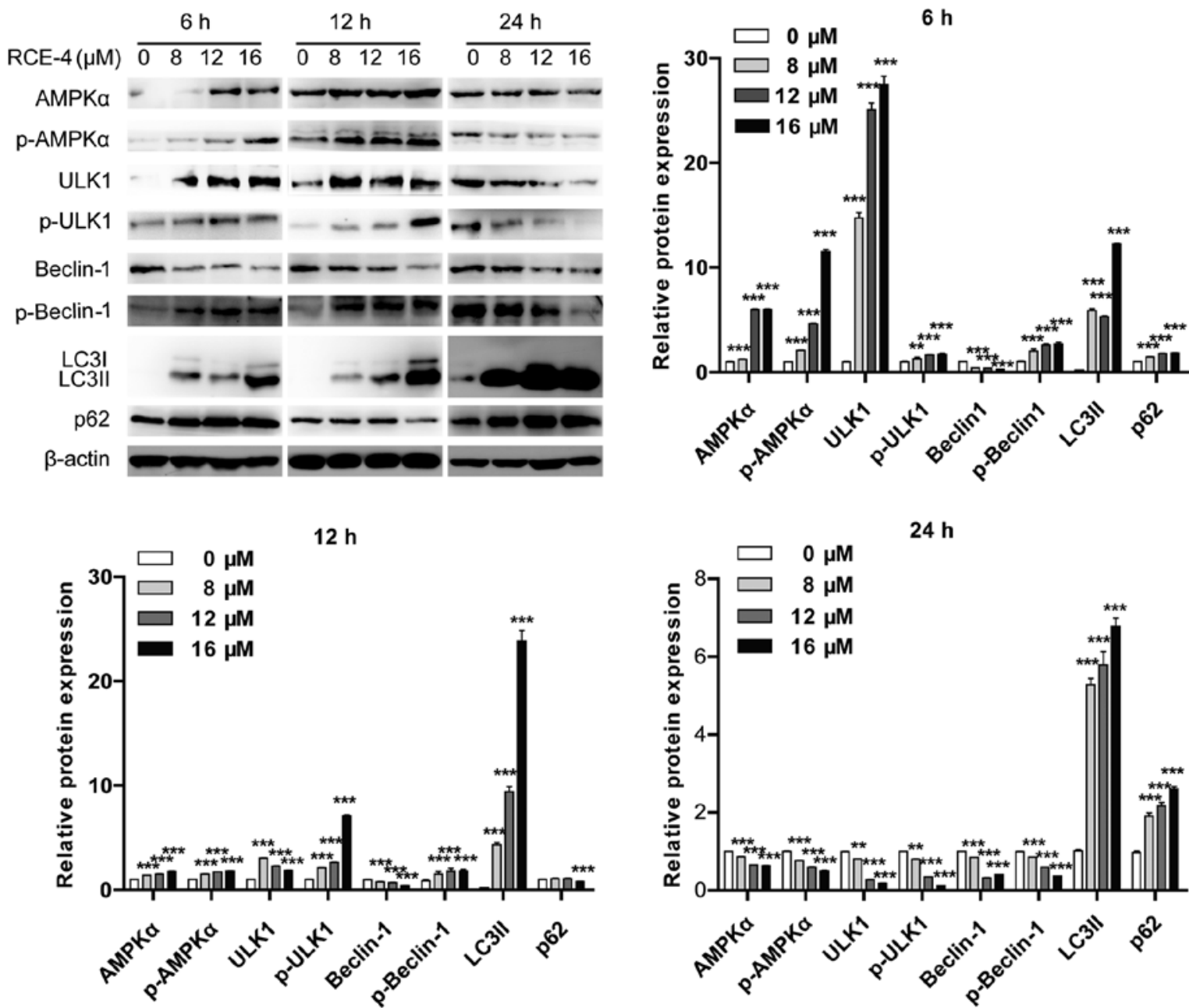

Figure 3. Expression levels of autophagy- (ULK1, p-ULK1, Beclin-1, p-Beclin-1, LC3II and p62) and AMPK pathway-associated proteins (AMPK and p-AMPK) in CaSki cells following treatment with different concentrations of RCE-4 for 6, 12 or $24 \mathrm{~h}$. Bar graphs represent the western blot grey values, presented as the mean \pm standard deviation. ${ }^{* *} \mathrm{P}<0.01$ and ${ }^{* * *} \mathrm{P}<0.001$ vs. $0 \mu \mathrm{M}$ RCE-4 group. ULK1, serine/threonine-protein kinase ULK1; $\mathrm{p}-$, phosphorylated; LC3, microtubule-associated proteins 1A/1B light chain 3B; AMPK, AMP-activated protein kinase; p62, sequestosome 1; RCE-4, (1 $\beta, 3 \beta, 5 \beta, 25 \mathrm{~S})$-spirostan-1, 3-diol 1-[ $\alpha$-L-rhamnopyranosyl-( $1 \rightarrow 2)-\beta$-D-xylopyranoside].

The location and number of autophagosomes and autolysosomes in RCE-4-treated cells was visualized using LCFM after CaSki cell transfection with mRFP-GFP-LC3 adenoviruses. $\mathrm{mRFP}$ is stably present in autolysosomes in an acidic environment, while GFP fluorescence is easily quenched. The emission of red and green fluorescence from one cell was recorded and merged; consequently, yellow dots, indicating autophagosomes, and red dots, indicating autolysosomes, appear in the merged images. When CaSki cells were treated with RCE-4 at a concentration of $12 \mu \mathrm{M}$ for 6 or $12 \mathrm{~h}$, an increased percentage of red fluorescent dots in the combined images was observed compared with the control group, representing autophagy activation. Conversely, when treated for 24 or $30 \mathrm{~h}$, autophagy may have been inhibited, based on a decreased percentage of red fluorescent dots (Fig. 2B and C).

As indicated in Fig. 3, RCE-4 increased ULK1, p-ULK1, p-Beclin-1 and lipid-modified LC3 (LC3II) expression levels, and downregulated p62 protein expression levels when used for $12 \mathrm{~h}$, compared with the control group. There were no significant alterations between the expression levels of autophagy-associated proteins, with the exception of p62, in CaSki cells treated with RCE-4 for 6 and $12 \mathrm{~h}$. Upregulation of the p62 expression level may be due to a compensatory increase in the number of autophagosomes at the initial stages of autophagy (31). Furthermore, following treatment with RCE-4 for $24 \mathrm{~h}$, the expression levels of ULK1 and p-Beclin-1 were downregulated, and that of p62 was upregulated, which further supported the inhibition of autophagy after prolonged treatment. Notably, it was expected that RCE-4 would induce an upregulation in the expression levels of Beclin-1, as it promotes autophagy; however, Beclin-1 expression was not upregulated in the RCE-4-treated cells.

Conversely, the increase in the LC3II protein expression level may be due to increased autophagosome formation following autophagy activation, or incomplete autolysosome clearance. Following pretreatment with autophagy inhibitors 3-MA, Baf A1 or CQ for $6 \mathrm{~h}$, the LC3II expression level in the 3-MA+RCE-4 group was decreased, compared with that of the RCE-4 group. However, the LC3II expression levels of the Baf A1+RCE-4 and the CQ+RCE-4 groups were increased following autophagy inhibition (Fig. 4A). Differences in LC3II relative protein expression were analyzed via densitometric analysis and presented in Fig. 4B. These results support the hypothesis that RCE-4 increases autophagic flux rather than inhibits the degradation 
A

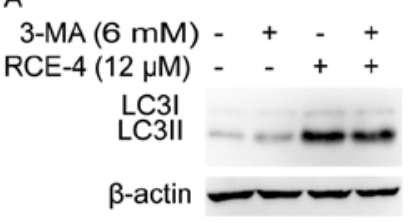

B

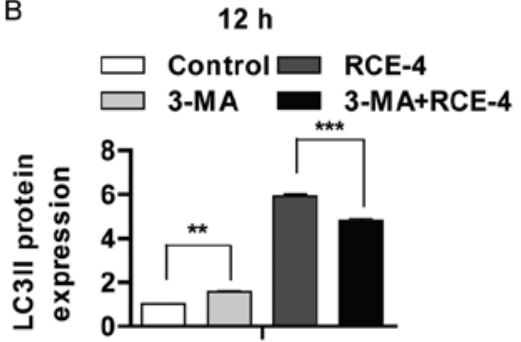

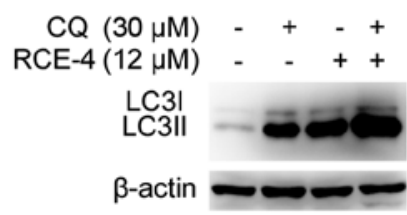

$12 \mathrm{~h}$

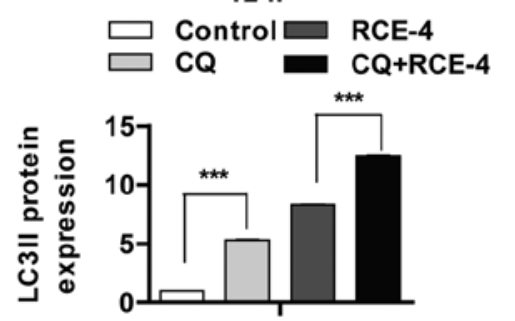

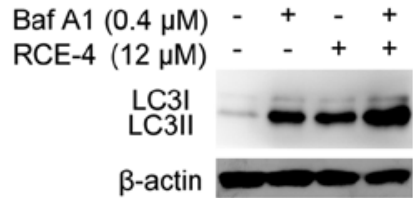

$12 \mathrm{~h}$

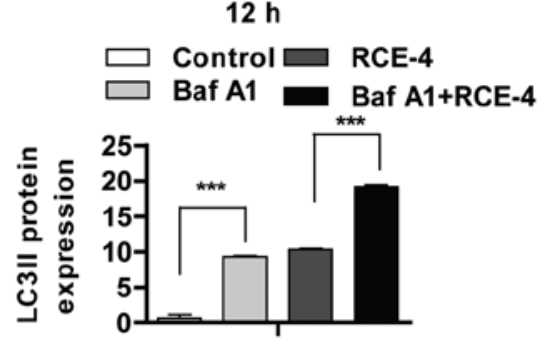

Figure 4. RCE-4 promotes the formation of autophagosomes in CaSki cells. (A) Effect of RCE-4 treatment for 12 h, with or without autophagy inhibitors, on the protein expression level of LC3II. (B) Bar graphs represent the western blot grey values, presented as the mean \pm standard deviation. ${ }^{* *} \mathrm{P}<0.01$ and ${ }^{* * *} \mathrm{P}<0.001$

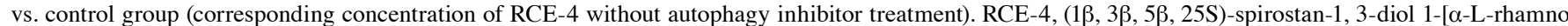
pyranosyl-(1 $\rightarrow 2)$ - $\beta$-D-xylopyranoside]; LC3, microtubule-associated proteins 1A/1B light chain 3B; LC3II, lipid-modified LC3; 3-MA, 3-methyladenine; CQ, chloroquine; Baf A1, bafilomycin A1.
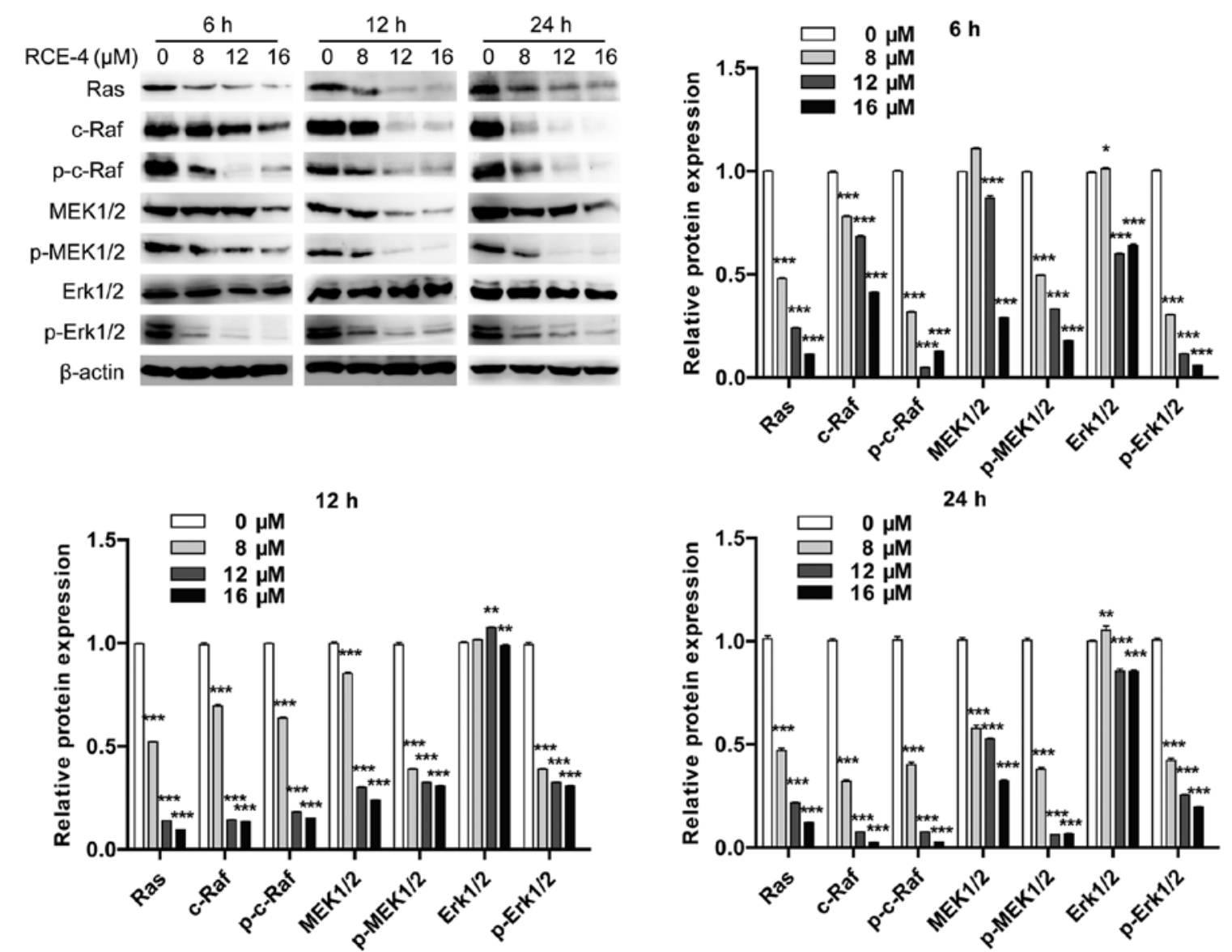

Figure 5. RCE-4 suppresses the Erk pathway signaling. Total proteins were collected from CaSki cells following treatment with various concentrations of RCE-4 $(0,8,12$ and $16 \mu \mathrm{M})$ for 6,12 or $24 \mathrm{~h}$. Expression levels of $\beta$-actin, Ras, total c-Raf, p-c-Raf, total MEK1/2, p-MEK1/2, total Erk1/2 and Erk1/2 were determined by western blot analysis. Bar graphs represent the western blot grey values, presented as the mean \pm standard deviation. ${ }^{*} \mathrm{P}<0.05$, ${ }^{* *} \mathrm{P}<0.01$ and ${ }^{* * * *} \mathrm{P}<0.001$ vs. 0 RCE-4 group. RCE-4, $(1 \beta, 3 \beta, 5 \beta, 25 \mathrm{~S})$-spirostan-1, 3-diol 1-[ $\alpha$-L-rhamnopyranosyl- $(1 \rightarrow 2)-\beta$-D-xylopyranoside]; MEK, mitogen-activated protein kinase kinase; Erk, extracellular signal-regulated kinase; p-, phosphorylated.

of autophagic components. In conclusion, RCE-4 induced autophagy after 6 and $12 \mathrm{~h}$, though such autophagy may be inhibited or ineffective after $24 \mathrm{~h}$ of treatment.
$R C E-4$ regulates autophagy by altering the protein expression level of mTOR. mTOR, which forms two distinct signaling complexes [mTOR complex (C)1 and mTORC2] by binding to 

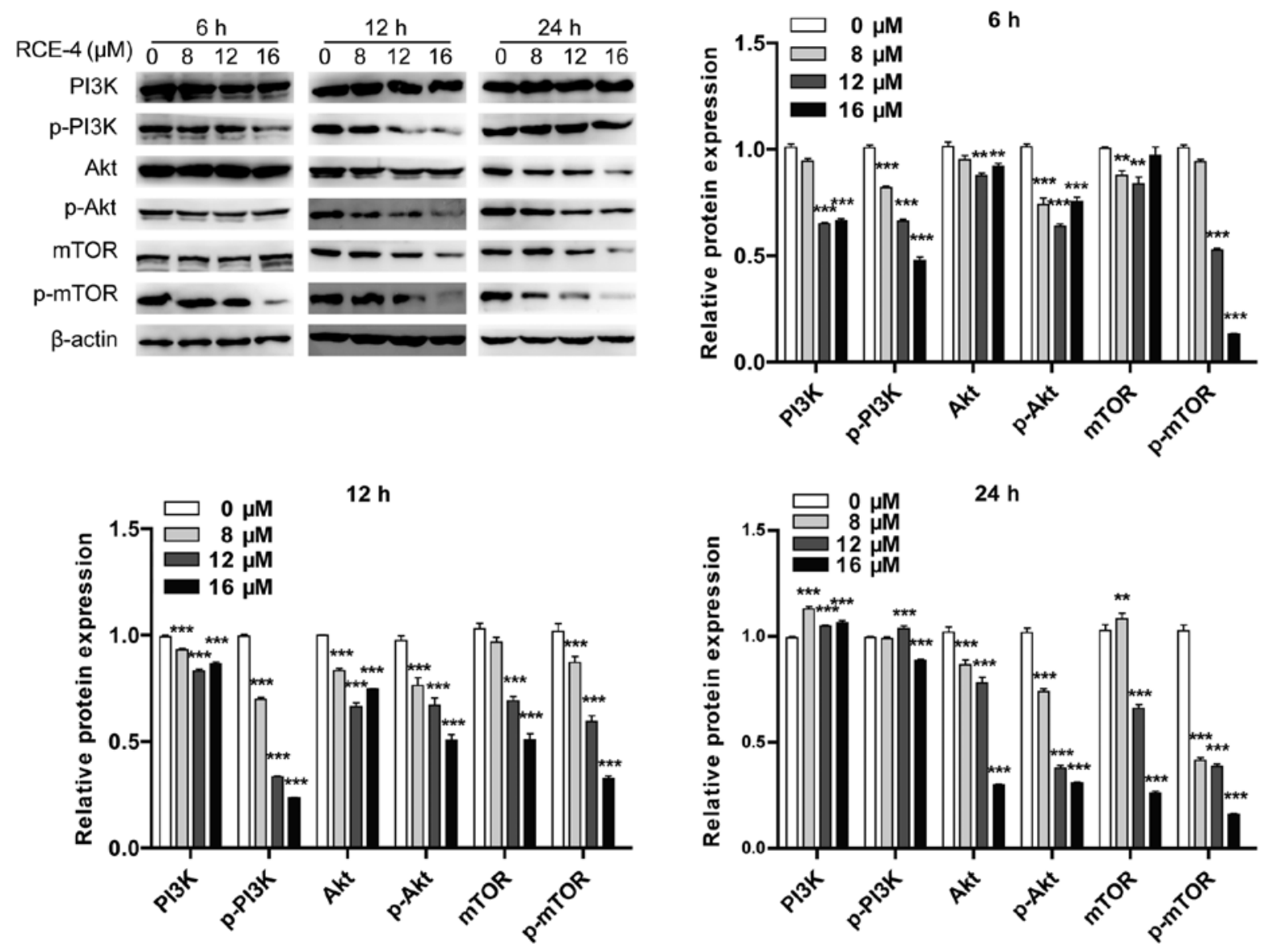

Figure 6. RCE-4 suppresses PI3K pathway signaling. Total proteins were collected from CaSki cells following treatment with various concentrations of RCE-4 $(0,8,12$ and $16 \mu \mathrm{M})$ for 6,12 or $24 \mathrm{~h}$. Expression levels of $\beta$-actin, total PI3K, p-PI3K, total Akt, p-Akt, total mTOR and p-mTOR were analyzed by western blot analysis. Bar graphs represent the western blot grey values, presented as the mean \pm standard deviation. ${ }^{* *} \mathrm{P}<0.01$ and ${ }^{* * *} \mathrm{P}<0.001$ vs. 0 RCE-4 group. RCE-4, $(1 \beta, 3 \beta, 5 \beta, 25 \mathrm{~S})$-spirostan-1, 3-diol 1-[ $\alpha$-L-rhamnopyranosyl-(1-2)- $\beta$-D-xylopyranoside]; $\mathrm{p}$-, phosphorylated.

multiple accompanying proteins, is a major regulator of cellular metabolism and serves a key role in autophagy regulation. The PI3K/AKT, Ras/Raf/MEK/Erk and AMPK pathways, activated by a high AMP/ATP ratio, are upstream signal cascades of mTOR (32). Western blot analysis revealed that AMPK and p-AMPK protein expression levels were upregulated at 6 and $12 \mathrm{~h}$, and downregulated at $24 \mathrm{~h}$ post-RCE-4 treatment in CaSki cells (Fig. 3). After a 24-h period, RCE-4 had also downregulated the expression of Ras, c-Raf, p-c-Raf, MEK1/2,p-MEK1/2 and p-Erk1/2 at all of the concentrations examined (Fig. 5). In addition, the expression levels of PI3K, $\mathrm{p}$-PI3K, p-Akt, and p-mTOR were inhibited at $6 \mathrm{~h}$ post-RCE- 4 treatment at concentrations of 12 and $16 \mu \mathrm{M}$; the protein expression levels of p-PI3K, p-Akt, mTOR and p-mTOR were suppressed at $12 \mathrm{~h}$ after post-RCE- 4 treatment; the protein expression levels of Akt, p-Akt, mTOR and p-mTOR were also suppressed at $24 \mathrm{~h}$ after post-RCE-4 treatment (Fig. 6).

\section{Discussion}

It is well known that cervical cancer is a serious health threat to the female population worldwide. Due to the negative effects of radiotherapy on ovarian function, chemotherapy as a treatment for cervical cancer has become increasingly valued by clinicians (33). RCE-4, a potential chemotherapeutic drug for cervical cancer, was demonstrated to induce mitochondria-mediated apoptosis $(5,9)$. However, not all apoptosis-inducing agents are effective in complex in vivo systems; for example, it has been reported that have demonstrated that the tumor necrosis factor-associated apoptosis-inducing ligand was unable to initiate apoptosis in tumor cells due to compensatory inhibition by autophagy $(34,35)$.

Autophagy and apoptosis may determine the fate of cells through cooperative or competitive pathways, depending on which process predominates (36). The aim of the present study was to assess the role of RCE-4-induced autophagy in proliferation inhibition, and the level and duration of autophagy in the CaSki cervical cancer cell line. To improve the understanding of the anti-tumor mechanisms induced by RCE-4, the molecular mechanisms of RCE-4-induced autophagy and the specific cellular targets or signaling pathways associated with its anti-proliferative activity, were additionally elucidated. It was demonstrated that RCE- 4 was potently cytotoxic to CaSki cells, which was characterized by decreased levels of cell proliferation and survival. Furthermore, the use of autophagy inhibitors enhanced proliferative inhibition following RCE-4 treatment, suggesting that autophagy promoted cell survival (37). In addition, it has been reported that have also demonstrated that autophagy inhibitors restrict the pro-survival function of autophagy, restore treatment sensitivity and promote tumor cell death (38-40). This indicates that RCE-4, combined with autophagy inhibitors, may be a promising strategy for cervical 
cancer treatment. Furthermore, the results of the present study support the role of RCE-4-induced autophagy and its contribution to the formation of chemoresistance.

Autophagy is a fundamental process of energy metabolism, where autophagosomes encapsulate dysfunctional cellular components, fuse with lysosomes to form autolysosomes, and facilitate degradation. Several important autophagy-associated proteins, including LC3II and p62, are involved in this process. LC3II, a classical autophagosomal marker, is located on the inner and outer membranes of autophagosomes and is often used to indicate autophagy. p62, which is inversely associated with selective autophagic degradation, serves as a bridge between LC3II and the ubiquitinated substrate to be degraded (41). Therefore, p62 and LC3II expression levels are important indicators of autophagy flux.

In the present study, it was determined that RCE-4 induced autophagy in CaSki cells. CYTO-ID staining revealed an increase in the number of autophagosomes in stained cells following an exposure period of 6, 12 or $24 \mathrm{~h}$. Furthermore, using mRFP-GFP-LC3 adenoviruses and LCFM an increase in autophagosome and autolysosome formation in CaSki cells following RCE-4 treatment was revealed. These data were supported by the results from the western blot analysis, which indicated that p62 was downregulated and LC3II was upregulated after $12 \mathrm{~h}$ of RCE-4 treatment. Furthermore, the use of 3 autophagy inhibitors (3-MA, CQ and Baf A1) with different targeting effects altered the expression level of LC3II following $12 \mathrm{~h}$ of RCE-4 treatment. LC3II was downregulated in the 3-MA+RCE-4 group, but upregulated in the CQ+RCE-4 and Baf A1+RCE-4 groups, compared with the RCE-4 control group. These results indicated that the increase in LC3II protein expression level was due to increased autophagosome formation following autophagy activation, which was consistent with harmine-induced autophagy (42). To summarize, the present study demonstrated that RCE-4 induced autophagy and the formation of autolysosomes, which were subsequently degraded.

Growth factors activate downstream signaling cascades, including the PI3K and ERK pathways, and thereby suppress the tuberous sclerosis (TSC) tumor suppressor complex TSC1/TSC2. As a GTPase-activating protein, the TSC complex is the most critical upstream negative regulator of mTORC1 (43). By contrast, AMPK activates TSC complexes under stress conditions, indirectly inhibiting the activity of mTORC1. AMPK also directly suppresses mTORC1 by phosphorylating regulatory-associated protein of mTOR, which itself binds to mTOR (44). In addition, mTORC1 not only suppresses autophagy by inhibiting ULK1/2 and vacuolar protein sorting-34 complex, but also decreases the transcriptional activity of lysosomal and autophagy-associated genes by phosphorylating transcription factor EB (32). mTORC1 and AMPK are upstream signaling components of ULK1, an autophagy-initiating kinase, and autophagy is regulated by the kinase activities of AMPK, mTOR and ULK1 (45). A previous study suggested that various AMPK and mTORC1-dependent phosphorylation events overlapped at ULK1 (20), indicating that both AMPK and mTORC1 tightly controlled the function of ULK1 through protein phosphorylation. To enhance phosphatidylinositol 3-kinase catalytic subunit type 3 (PIK3C3) activity in complexes containing $\mathrm{UV}$ radiation resistance-associated gene protein (UVRAG) or
Beclin-1-associated autophagy-related key regulator (ATG14), AMPK phosphorylates Beclin-1 at Ser91 and Ser94; these ATG14- or UVRAG-containing complexes are involved in autophagy initiation. Activation of PIK3C3 complexes containing ATG14 by Beclin-1 phosphorylation was demonstrated to be necessary for autophagy induction (46). In the present study, it was demonstrated that following $12 \mathrm{~h}$ of RCE-4 treatment, the expression levels of AMPK, p-AMPK, ULK1, p-ULK1, p-Beclin-1 and LC3II were upregulated, and mTOR and p-mTOR expression levels were downregulated, indicating that autophagy had been initiated. It was also revealed that when treated with RCE-4, the expression levels of p-PI3K, p-Akt, mTOR, p-mTOR, Ras, c-Raf, p-c-Raf, MEK1/2, p-MEK1/2 and p-Erk1/2 were decreased. Therefore, RCE-4-induced autophagy was concluded to be closely associated with the AMPK pathway, and the dual blockade of the ERK and PI3K pathways.

Notably, in the present study, after $24 \mathrm{~h}$ of treatment, RCE-4-mediated autophagy was inhibited, or RCE-4 may have prevented normal lysosomal functioning, which was indicated by a percentage decrease in autolysosome staining, the downregulation of AMPK,p-AMPK, ULK1,p-ULK1 and p-Beclin-1, and the upregulation of p62 expression levels. Wang et al (29) and Feng et al (30) reported that the inhibition of autophagy was associated with a decreased percentage of autolysosomes and an upregulated expression of p62. p-AMPK, p-ULK1 and p-Beclin-1 exhibited similar time- and concentration-dependent changes. This is supported by the previous observation of direct phosphorylation and activation of ULK1 and Beclin-1 kinases by AMPK (47). Although the results from the present study demonstrated that the activation of autophagy was regulated by the AMPK pathway, the RCE-4-induced protective autophagy in CaSki cells was not conducive to the anti-cervical cancer effect of RCE-4; however, autophagy may have been inhibited by prolonged treatment times. Furthermore, the downregulation of Beclin-1 levels indicated that crosstalk and feedback mechanisms between RCE-4-induced apoptosis and autophagy may be involved (48).

Activation of the ERK and PI3K pathways promotes tumor cell proliferation and inhibits apoptosis $(3,18,25)$. RCE-4 has a stronger inhibitory effect on CaSki cells by inducing the dual blockade of the Ras/Raf/MEK/Erk and PI3K/Akt/mTOR pathways, compared with a single pathway inhibitor. These pathways are the most frequently dysregulated kinase cascades in human cancer (49), and both PI3K/Akt/mTOR and Ras/Raf/MEK/Erk cascade inhibitors have been studied for their possible chemotherapeutic use (50). The present study suggested that the cytotoxicity of RCE-4 was closely associated with the suppression of PI3K/AKT and Ras/Raf/MEK/Erk signaling. However, it is unclear how RCE-4 mediates both pathways, and this requires further investigation.

To conclude, the present study revealed that RCE-4 inhibited the proliferation of cervical cancer cells, and activated protective autophagy. In addition, RCE-4 activated the AMPK pathway, and inhibited PI3K and ERK signaling pathways by regulating the associated proteins involved in autophagy. These data may provide novel insights into the molecular mechanisms of the potential anti-cervical cancer effects of RCE-4, and assist in developing this potential candidate for cervical cancer treatment. 


\section{Acknowledgements}

The authors would like to the Medical College of China Three Gorges University for the provision of experimental equipment.

\section{Funding}

The present study was funded by the National Natural Science Foundation of China (grant no. 81773952) and the Traditional Chinese Medicine Research Foundation of the Health Commission of Hubei Province (grant no. ZY2019M028).

\section{Availability of data and materials}

The datasets used and/or analyzed during the current study are available from the corresponding author upon request.

\section{Authors' contributions}

JFC conceived and designed the research, supplied reagents, materials, analysis tools and designed certain experimental methods. WXiang performed the experiments and wrote the manuscript. GLJ, XFX and WXi collected Reineckia carnea herbs in the field and completed their extraction and separation. FC and JZW purified and identified RCE-4 compounds. RJZ, LT and SJT assisted in the analysis of data and provided key commentary. All authors read the results and approved the final version of the manuscript.

\section{Ethics approval and consent to participate}

Not applicable.

\section{Patient consent for publication}

Not applicable.

\section{Competing interests}

The authors declare that they have no competing interests.

\section{References}

1. Jiang F, Zhou JY, Zhang D, Liu MH and Chen YG: Artesunate induces apoptosis and autophagy in HCT116 colon cancer cells, and autophagy inhibition enhances the artesunate induced apoptosis. Int J Mol Med 42: 1295-1304, 2018.

2. García-Zepeda SP, García-Villa E, Díaz-Chávez J, Hernández-Pando R and Gariglio P: Resveratrol induces cell death in cervical cancer cells through apoptosis and autophagy. Eur J Cancer Prev 22: 577-584, 2013.

3. Xu T, Pang Q, Wang Y and Yan X: Betulinic acid induces apoptosis by regulating PI3K/Akt signaling and mitochondrial pathways in human cervical cancer cells. Int J Mol Med 40 1669-1678, 2017.

4. Sy LK, Yan SC,Lok CN, Man RY and Che CM:Timosaponin A-III induces autophagy preceding mitochondria-mediated apoptosis in HeLa cancer cells. Cancer Res 68: 10229-10237, 2008.

5. Bai C, Yang X, Zou K, He H, Wang J, Qin H, Yu X, Liu C, Zheng J, Cheng F, et al: Anti-proliferative effect of RCE-4 from Reineckia carnea on human cervical cancer HeLa cells by inhibiting the $\mathrm{PI} 3 \mathrm{~K} / \mathrm{Akt} / \mathrm{mTOR}$ signaling pathway and NF- $\mathrm{BB}$ activation. Naunyn Schmiedebergs Arch Pharmacol 389: 573-584, 2016.

6. Wang Q, Hou Q, Guo Z, Zou K, Xue Y, Huang N, Cheng F and Zhou Y: Three new steroidal glycosides from roots of Reineckia carnea. Nat Prod Res 27: 85-92, 2013.
7. Tang Y, Li N, Duan JA and Tao W: Structure, bioactivity, and chemical synthesis of OSW-1 and other steroidal glycosides in the genus Ornithogalum. Chem Rev 113: 5480-5514, 2013.

8. Fu XJ, Zou K, Wang GP and Zhang X: Anti-inflammatory effect and mechanism of ethyl acetate extract from Reineckia carnea. Lishizhen Med Mater Med Res 24: 822-825, 2013 (In Chinese).

9. Wang G, Huang W, He H, Fu X, Wang J, Zou K and Chen J: Growth inhibition and apoptosis-inducing effect on human cancer cells by RCE-4, a spirostanol saponin derivative from natural medicines. Int J Mol Med 31: 219-224, 2013.

10. Yang XJ, Bai CH, Zou K, He HB, Yu XQ, Qin HL, Zhang YF and Wang JZ: Steroidal saponin RCE-4 from Reineckia carnea (Andr.) Kunth inhibits growth of human cervical cancer xenograft in nude mice. J Third Mil Med Univ 38: 476-482, 2016 (In Chinese).

11. Chaabane W, User SD, El-Gazzah M, Jaksik R, Sajjadi E, Rzeszowska-Wolny J and Los MJ: Autophagy, apoptosis, mitoptosis and necrosis: Interdependence between those pathways and effects on cancer. Arch Immunol Ther Exp (Warsz) 61: 43-58, 2013.

12. Levine B and Kroemer G: Autophagy in the pathogenesis of disease. Cell 132: 27-42, 2008.

13. Pham DC, Chang YC, Lin SR, Fuh YM, Tsai MJ and Weng CF: FAK and S6K1 inhibitor, neferine, dually induces autophagy and apoptosis in human neuroblastoma cells. Molecules 23: 3110-3125, 2018

14. Russo M and Russo GL: Autophagy inducers in cancer. Biochem Pharmacol 153: 51-61, 2018.

15. Eisenberg-Lerner A, Bialik S, Simon HU and Kimchi A: Life and death partners: Apoptosis, autophagy and the cross-talk between them. Cell Death Differ 16: 966-975, 2009.

16. White E and DiPaola RS: The double-edged sword of autophagy modulation in cancer. Clin Cancer Res 15: 5308-5316, 2009.

17. Ouyang L, Shi Z, Zhao S, Wang FT, Zhou TT, Liu B and Bao JK: Programmed cell death pathways in cancer: A review of apoptosis, autophagy and programmed necrosis. Cell Prolif 45: 487-498, 2012.

18. De Luca A, Maiello MR, D'Alessio A, Pergameno $M$ and Normanno N: The RAS/RAF/MEK/ERK and the PI3K/AKT signalling pathways: Role in cancer pathogenesis and implications for therapeutic approaches. Expert Opin Ther Targets 16 (Suppl 2): S17-S27, 2012.

19. Kim J, Kundu M, Viollet B and Guan KL: AMPK and mTOR regulate autophagy through direct phosphorylation of Ulk1. Nat Cell Biol 13: 132-141, 2011.

20. Shang L, Chen S, Du F, Li S, Zhao L and Wang X: Nutrient starvation elicits an acute autophagic response mediated by Ulk1 dephosphorylation and its subsequent dissociation from AMPK. Proc Natl Acad Sci USA 108: 4788-4793, 2011.

21. Song L, Wang Z, Wang Y, Guo D, Yang J, Chen L and Tan N: Natural cyclopeptide RA-XII, a new autophagy inhibitor, suppresses protective autophagy for enhancing apoptosis through AMPK/mTOR/P70S6K pathways in HepG2 cells. Molecules 22: 1934-1950, 2017.

22. Vakifahmetoglu-Norberg H, Xia HG and Yuan J: Pharmacologic agents targeting autophagy. J Clin Invest 125: 5-13, 2015.

23. Sooro MA, Zhang $\mathrm{N}$ and Zhang P: Targeting EGFR-mediated autophagy as a potential strategy for cancer therapy. Int $\mathrm{J}$ Cancer 143: 2116-2125, 2018.

24. Chappell WH, Steelman LS, Long JM, Kempf RC, Abrams SL, Franklin RA, Bäsecke J, Stivala F, Donia M, Fagone P, et al: Ras/Raf/MEK/ERK and PI3K/PTEN/Akt/mTOR inhibitors: Rationale and importance to inhibiting these pathways in human health. Oncotarget 2: 135-164, 2011.

25. McCubrey JA, Steelman LS, Chappell WH, Abrams SL, Wong EW, Chang F, Lehmann B, Terrian DM, Milella M, Tafuri A, et al: Roles of the Raf/MEK/ERK pathway in cell growth, malignant transformation and drug resistance. Biochim Biophys Acta 1773: 1263-1284, 2007.

26. Normanno N, De Luca A, Maiello MR, Campiglio M, Napolitano M, Mancino M, Carotenuto A, Viglietto G and Menard S: The MEK/MAPK pathway is involved in the resistance of breast cancer cells to the EGFR tyrosine kinase inhibitor gefitinib. J Cell Physiol 207: 420-427, 2006.

27. Serra V, Scaltriti M, Prudkin L, Eichhorn PJA, Ibrahim YH, Chandarlapaty S, Markman B, Rodriguez O, Guzman M, Rodriguez S, et al: PI3K inhibition results in enhanced HER signaling and acquired ERK dependency in HER2-overexpressing breast cancer. Oncogene 30: 2547-2557, 2011.

28. Mqoco T and Joubert A: 2-Methoxyestradiol-bis-sulphamate induces apoptosis and autophagy in an oesophageal carcinoma (SNO) cell line. Biomed Res-India 23: 469-474, 2012. 
29. Wang Y, Nie H, Zhao X, Qin Y and Gong X: Bicyclol induces cell cycle arrest and autophagy in HepG2 human hepatocellular carcinoma cells through the PI3K/AKT and Ras/Raf/MEK/ERK pathways. BMC Cancer 16: 742-757, 2016.

30. Feng X, Zhou J, Li J, Hou X, Li L, Chen Y, Fu S, Zhou L, Li C and Lei Y: Tubeimoside I induces accumulation of impaired autophagolysosome against cervical cancer cells by both initiating autophagy and inhibiting lysosomal function. Cell Death Dis 9: 1117-1133, 2018.

31. Zheng Q, Su H, Ranek MJ and Wang X: Autophagy and p62 in cardiac proteinopathy. Circ Res 109: 296-308, 2011.

32. Kim YC and Guan KL: mTOR: A pharmacologic target for autophagy regulation. J Clin Invest 125: 25-32, 2015.

33. Jemal A, Simard EP, Dorell C, Noone AM, Markowitz LE, Kohler B, Eheman C, Saraiya M, Bandi P, Saslow D, et al: Annual Report to the Nation on the Status of Cancer, 1975-2009, featuring the burden and trends in human papillomavirus (HPV)-associated cancers and HPV vaccination coverage levels. J Natl Cancer Inst 105: 175-201, 2013.

34. Nazim UM and Park SY: Attenuation of autophagy flux by 6-shogaol sensitizes human liver cancer cells to TRAIL-induced apoptosis via p53 and ROS. Int J Mol Med 43: 701-708, 2019.

35. Thorburn A, Behbakht $\mathrm{K}$ and Ford H: TRAIL receptor-targeted therapeutics: Resistance mechanisms and strategies to avoid them. Drug Resist Updat 11: 17-24, 2008.

36. Sun Y, Liu JH, Jin L, Lin SM, Yang Y, Sui YX and Shi H: Over-expression of the Beclin1 gene upregulates chemosensitivity to anti-cancer drugs by enhancing therapy-induced apoptosis in cervix squamous carcinoma CaSki cells. Cancer Lett 294: 204-210, 2010.

37. Lima RT, Sousa D, Paiva AM, Palmeira A, Barbosa J, Pedro M, Pinto MM, Sousa E and Vasconcelos MH: Modulation of Autophagy by a Thioxanthone Decreases the Viability of Melanoma Cells. Molecules 21: 1343-1358, 2016.

38. Maycotte P, Aryal S, Cummings CT, Thorburn J, Morgan MJ and Thorburn A: Chloroquine sensitizes breast cancer cells to chemotherapy independent of autophagy. Autophagy 8: 200-212, 2012.

39. Rao R, Balusu R, Fiskus W, Mudunuru U, Venkannagari S, Chauhan L, Smith JE, Hembruff SL, Ha K, Atadja P, et al: Combination of pan-histone deacetylase inhibitor and autophagy inhibitor exerts superior efficacy against triple-negative human breast cancer cells. Mol Cancer Ther 11: 973-983, 2012.
40. Palmeira dos Santos C, Pereira GJS, Barbosa CMV, Jurkiewicz A, Smaili SS and Bincoletto C: Comparative study of autophagy inhibition by 3MA and CQ on Cytarabine induced death of leukaemia cells. J Cancer Res Clin Oncol 140: 909-920, 2014.

41. Lin YT, Wang HC, Hsu YC, Cho CL, Yang MY and Chien CY: Capsaicin induces autophagy and apoptosis in human nasopharyngeal carcinoma cells by downregulating the $\mathrm{PI} 3 \mathrm{~K} / \mathrm{AKT} / \mathrm{mTOR}$ Tathway. Int J Mol Sci 18: 1343-1359, 2017.

42. Zou N, Wei Y, Li F, Yang Y, Cheng X and Wang C: The inhibitory effects of compound Muniziqi granule against B16 cells and harmine induced autophagy and apoptosis by inhibiting Akt/mTOR pathway. BMC Complement Altern Med 17: 517-528, 2017.

43. Inoki K, Zhu T and Guan KL: TSC2 mediates cellular energy response to control cell growth and survival. Cell 115: 577-590, 2003.

44. Gwinn DM, Shackelford DB, Egan DF, Mihaylova MM, Mery A, Vasquez DS, Turk BE and Shaw RJ: AMPK phosphorylation of raptor mediates a metabolic checkpoint. Mol Cell 30: 214-226, 2008.

45. Dunlop EA and Tee AR: mTOR and autophagy: A dynamic relationship governed by nutrients and energy. Semin Cell Dev Biol 36: 121-129, 2014

46. Kim J, Kim YC, Fang C, Russell RC, Kim JH, Fan W, Liu R, Zhong Q and Guan KL: Differential regulation of distinct Vps34 complexes by AMPK in nutrient stress and autophagy. Cell 152: 290-303, 2013.

47. Russell RC, Yuan HX and Guan KL: Autophagy regulation by nutrient signaling. Cell Res 24: 42-57, 2014.

48. Pattingre S, Tassa A, Qu X, Garuti R, Liang XH, Mizushima N, Packer M, Schneider MD and Levine B: Bcl-2 antiapoptotic proteins inhibit Beclin 1-dependent autophagy. Cell 122: 927-939, 2005

49. Su CC: Tanshinone IIA can inhibit MiaPaCa-2 human pancreatic cancer cells by dual blockade of the Ras/Raf/MEK/ERK and PI3K/AKT/mTOR pathways. Oncol Rep 40: 3102-3111, 2018.

50. McCubrey JA, Steelman LS, Chappell WH, Abrams SL, Montalto G, Cervello M, Nicoletti F, Fagone P, Malaponte G, Mazzarino MC, et al: Mutations and deregulation of Ras/Raf/MEK/ERK and PI3K/PTEN/Akt/mTOR cascades which alter therapy response. Oncotarget 3: 954-987, 2012. 